\title{
A NEW DENOISING APPROACH FOR THE REMOVAL OF IMPULSE NOISE FROM COLOR IMAGES AND VIDEO SEQUENCES
}

\author{
ARUMUGAM RAJAMANI $^{\bowtie, 1}$, VELLINGIRI KRISHNAVENI ${ }^{2}$, HABEEBRAKUMAN WASSIM FEROSE ${ }^{3}$ \\ AND MOHAN KALAIKAMAL ${ }^{3}$ \\ ${ }^{1}$ PSG Polytechnic College; ${ }^{2,3}$ PSG College of Technology; ${ }^{1,2,3}$ Tamilnadu, India \\ e-mail: hod@dec.psgtech.ac.in; vk@ece.psgtech.ac.in; wassimferose.h@gmail.com;kalaikamal.psg@gmail.com \\ (Received June 12, 2012; revised September 6, 2012; accepted November 18, 2012)
}

\begin{abstract}
In this paper a novel Lone Diagonal Sorting (LDS) algorithm for denoising color images and videos corrupted with salt and pepper noise is proposed. The proposed lone diagonal sorting algorithm uses diagonal sorting alone for denoising of impulse noise. The algorithm has been implemented and tested for various color images and video signals and appreciable performance in terms of PSNR, MSE and SSIM is obtained. Our algorithm has been compared with other standard algorithms. A drastic improvement in the computational time has been achieved without compromising much on the visual quality after reconstruction.
\end{abstract}

Keywords: color image, lone diagonal sorting, mean square error, peak signal to noise ratio, salt and pepper noise.

\section{INTRODUCTION}

A color image is a digital image that includes color information for each pixel. For visually acceptable results, it is necessary to provide three samples or color channels for each pixel, which are interpreted as coordinates in some color space. The RGB color space is commonly used in computer displays, but other spaces such as YCbCr and HSV are often used in other contexts (Pratt, 1978). The RGB color model is basically an additive color model in which red, green, and blue light is added together in various ways to reproduce a broad array of colors (Gonzalez and Woods, 2008). On the other hand, video is the technology of electronically capturing, recording, processing, storing, transmitting, and reconstructing a sequence of still images representing scenes in motion (Aiswarya et al., 2010). The video signals are hence continuous images and these individual images are referred to as the frames. The frame rate is an important terminology concerning the video signals and is defined as the number of still pictures per unit time of video and the minimum frame rate to achieve the illusion of a moving image is about fifteen frames per second.

Our paper deals with the image quality enhancement of color images following RGB color space and the video sequences that are affected with impulse noise. As low-end imaging devices, such as web-ca- meras and mobile phones become essential, there is an ever more need for reliable digital image and video enhancement technologies to improve their outputs. Noise is a dominant factor that degrades image quality (Pitas and Venetsanopoulous, 1990).

Impulse noise or salt and pepper noise creeps into images in situations where quick transients such as faulty switching take place and also can occur due to a random bit error in a communication channel. The pixel values (0 or 255) will be considered as noise, in reality it will occur due to bit error or during quick transients. Other than 0 or 255 all values are non noisy according to this algorithm.

\section{IMPULSE NOISE IN IMAGES AND VIDEOS AND ITS FILTERING}

Images and video signals are often corrupted by additive noise and/or motion blur mostly during acquisition and transmission. Consequently, denoising these signals in order to remove the effect of noise is highly desired (Astola and Kuosmanen, 1997). Denoising of color images and video signals is highly desirable in order to enhance the overall perceptual quality, increase compression effectiveness, facilitate transmission bandwidth reduction, and facilitate accuracy in processes like feature extraction and pattern recognition that might be involved. 
This paper aims at developing a robust video denoising algorithm capable of removing salt and pepper noise from the image sequences. Salt and pepper noise is a typical type of noise in images and videos. This noise is caused by errors in data transmission or faulty memory location in hardware. It takes the form of randomly occurring white and black pixels. Usual and effective noise reduction method for this type of noise involves the usage of median filter. This algorithm proves quite effective when the noise level is low. But at higher noise densities, standard median filters often exhibit blurring for large window sizes and insufficient noise suppression for small window sizes (Gallaghera and Wise, 1981; Nodes and Gallagher, 1987).

The objective of filtering is to remove the impulses so that the noise free image is fully recovered with minimum signal distortion. Noise removal can be achieved by using a number of existing linear filtering techniques which are popular because of their mathematical simplicity and the existence of the unifying linear system theory (JeaKo and Lee, 1991). Though median filters remove impulse noise without damaging the edges, most of them operate uniformly across the image and thus tend to modify both noise and noise-free pixels (Luo, 2006a,b). Ideally, the filtering should be applied only to corrupted pixels while leaving uncorrupted pixels intact. Therefore, a noise-detection process to discriminate between uncorrupted pixels and the corrupted pixels prior to applying nonlinear filtering is highly desirable (Chan et al., 2005). The discrimination between the noisy and noise-free pixels can be easily done because the impulse noise pixels can take the maximum and minimum values in the dynamic range $(0,255)$ (Ratnababu et al., 2011).

The proposed algorithm uses just 3 comparisons per window through diagonal sorting and hence lower number of computations leading to robustness can be achieved. Earlier algorithms use combinations of row, column and diagonal sorting which increases computational and hardware complexity. Also reduction in the operating speed due to more computations is observed. But the Lone Diagonal Sorting (LDS) algorithm proposed in this paper uses diagonal sorting alone to obtain appreciable results in terms of computational complexity and visual appearance than existing algorithms.

\section{PROPOSED LONE DIAGONAL SORTING ALGORITHM FOR COLOR IMAGES AND VIDEO SEQUENCES}

\section{Algorithm for color images}

The algorithm for lone diagonal sorting for color images is described below. For each channel of the color image (red, green and blue), the following is implemented.

STEP 1: First the "red" array is selected among three channels.

STEP 2: A 2D window of size 3x3 in a 512x512 array is chosen. Aij is assumed as the pixel being processed.

\begin{tabular}{||c||c|c|c||}
\hline 0 & 25 & 37 & 24 \\
\hline 34 & 27 & 29 & 34 \\
\hline 22 & 24 & 255 & 29 \\
\hline 25 & 28 & 29 & 26 \\
\hline
\end{tabular}

Fig. 1. Selection of sliding window (3x3).

STEP 3: The leading diagonal elements (only three elements in this case) of the window size $3 \times 3$ is selected and sorted. Assume that the diagonal pixels being processed are $\mathrm{D}_{11}, \mathrm{D}_{22}$ and $\mathrm{D}_{33}$ (for example 0, 27 and 255 as given in the (Fig. 2).

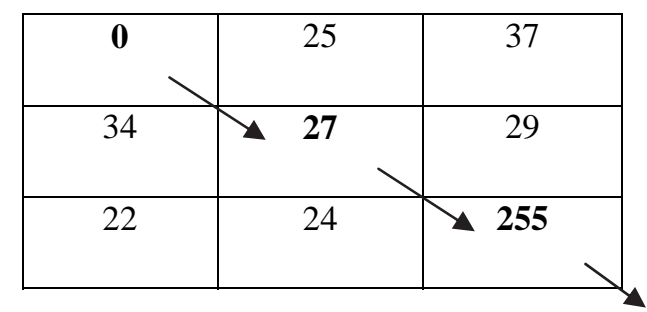

Fig. 2. Original window (3x3) with noises at $D_{11} \& D_{33}$.

If the diagonal element $0<\mathrm{D}_{\mathrm{ij}}<255$, then it is classified as a noise free pixel, and is left unchanged. If the diagonal element $\mathrm{D}_{\mathrm{ij}}=0$ or 255 then it is classified as noisy pixel and the replacing technique is applied since the proposed algorithm aims to remove the noisy pixels such as salt(255) and pepper(0) in an effective manner.

STEP 4: Each diagonal pixel is checked for salt and pepper noise ( 0 or 255) and replaced with the either of the other values which are noise free. In the above case the $D_{11}$ is $0, D_{22}$ is 27 and $D_{33}$ is 255 . 
STEP 5: Sorting of the three diagonal elements is performed in the ascending order sorting result comes as $0,27,255$ and middle element called as median value (in this case 27) is replaced for the diagonal element $D_{11}$ and $D_{33}$ which are pepper and salt noises respectively. The advantages of the proposed algorithm at this point could be that it involves three comparisons only when compared to other sorting methods. Table 1 shows the comparison of sorting techniques. So hardware complexity is very much reduced and speed of operation can be increased.

Table 1. Comparative results of various sorting techniques.

\begin{tabular}{ll}
\hline Sorting techniques & $\begin{array}{l}\text { No. of comparisons required to } \\
\text { compute median of 3x3 } \\
\text { window }\end{array}$ \\
\hline Bubble sort & 36 \\
Shear sort & 18 \\
Lone diagonal sort & 3 \\
\hline
\end{tabular}

\begin{tabular}{|l|l|l|}
\hline 27 & 25 & 37 \\
\hline 34 & 27 & 29 \\
\hline 22 & 24 & 255 \\
\hline
\end{tabular}

Fig. 3. Window (3x3) with salt noise only at $D_{33}$.

\begin{tabular}{|l|l|l|}
\hline 27 & 25 & 37 \\
\hline 34 & 27 & 29 \\
\hline 22 & 24 & 27 \\
\hline
\end{tabular}

Fig. 4. Denoised window (3x3).

If all the three diagonal pixels are noisy, then the first pixel, $\mathrm{D}_{11}$ is replaced with a non-noisy 4-neighborhood pixel and the processing is immediately moved to STEP 6. The pixels, $\mathrm{D}_{22}$ and $\mathrm{D}_{33}$ would be processed in the subsequent vertical or horizontal windows.

STEP 6: Horizontal and vertical shift pulses are applied to move to the next $3 \times 3$ sliding window and STEP 3,4 and STEP 5 are repeated until processing is completed for the entire array.

STEP 7: A shift pulse is applied and the above pro- cesses (step1 to step6) are repeated subsequently for the "green" and "blue" 512x512 array. The same process is carried over for all three RGB arrays to denoise entire color image.

\section{Implementation for video sequence}

The video sequence is first converted into frames and consecutively frames into images. Then the proposed lone diagonal sorting algorithm is applied to the images which are separated from frames. After the filtering process, the frames are converted back to the original movie.

The algorithm is as follows:

STEP 1: The noisy video sequence affected with impulse noise is converted into .avi format, which is an uncompressed format and the individual frames are extracted from the video as follows in Matlab environment.

STEP 2: The extracted frames are then converted to images for further processing.

STEP 3: The noise affected images are denoised using the proposed LDS algorithm.

STEP 4: After completing the entire process, the denoised frames are finally reproduced back into the original movie.

\section{RESULTS}

The developed algorithm is tested using $512 \times 512$ images; Parrot (color), Barbara (color). The performance of the proposed algorithm is tested for all levels of noise corruption (up to 90\%). Each time the test image is corrupted by salt and pepper noise of different density ranging from $10 \%$ to $90 \%$ with an increment of $10 \%$ and tested for denoising. The results are promising and obtained in good reconstruction quality even at high level noise densities too and are hence shown in (Figs. 5-10). For video sequence denoising, the individual frames are extracted from the noise affected video sequences and the proposed algorithm is applied. After the noise removal, the frames are converted back to video sequences. The video frames which are corrupted by the noise density vary from $10 \%$ to $60 \%$ and its denoised results are shown in (Figs. 11,12). 


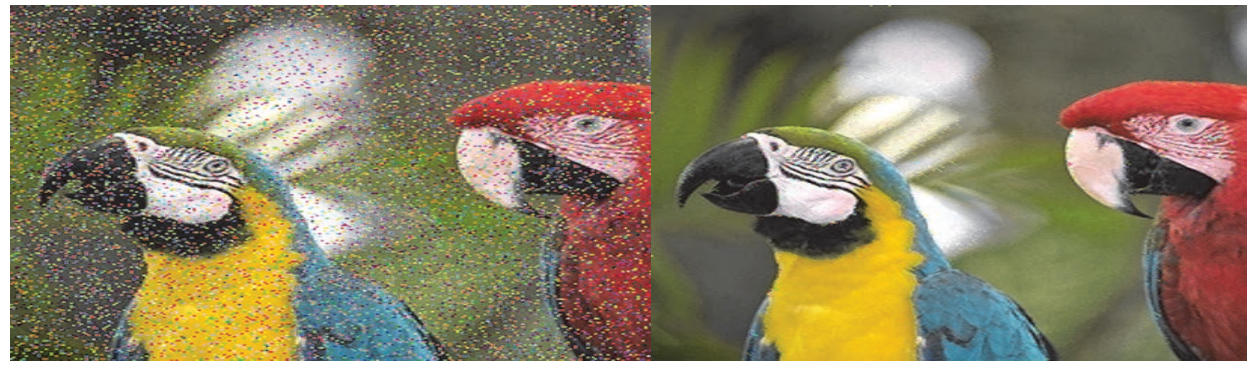

Fig. 5. Results obtained for the noise level (10\%) corrupted for the 512x512 parrot color image and its denoised image.

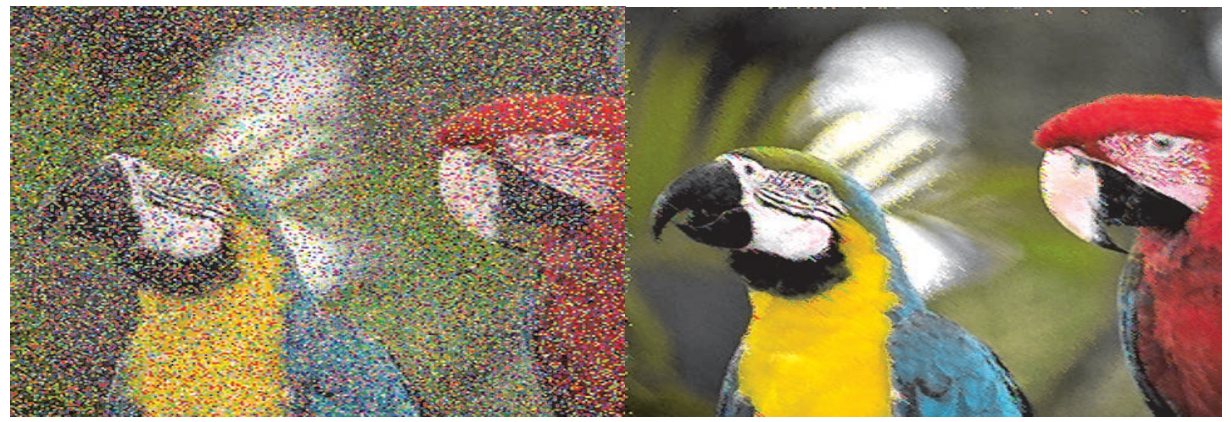

Fig. 6. Results obtained for the noise level (30\%) corrupted for the 512x512 parrot color image and its denoised image.

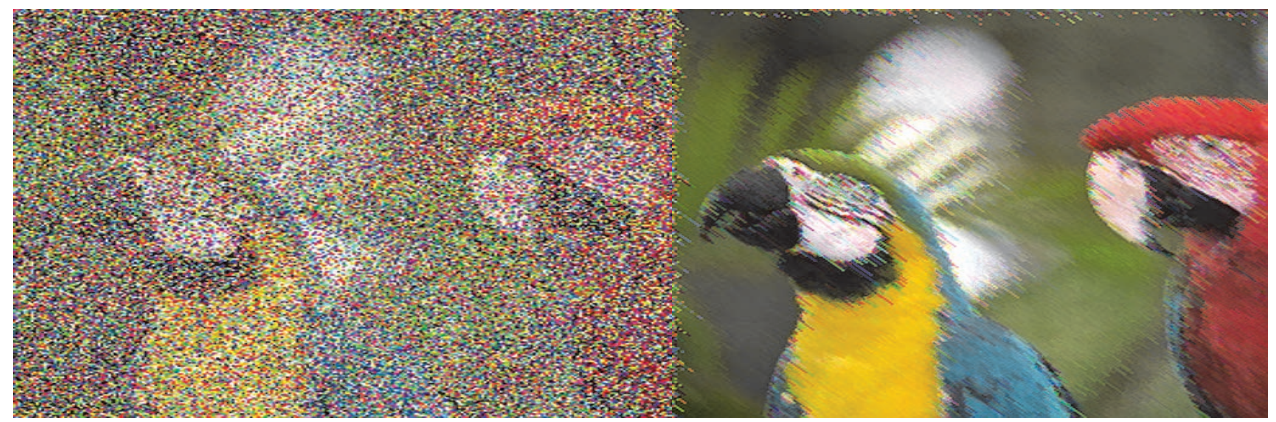

Fig. 7. Results obtained for the noise level (60\%) corrupted for the 512x512 parrot color image and its denoised image.

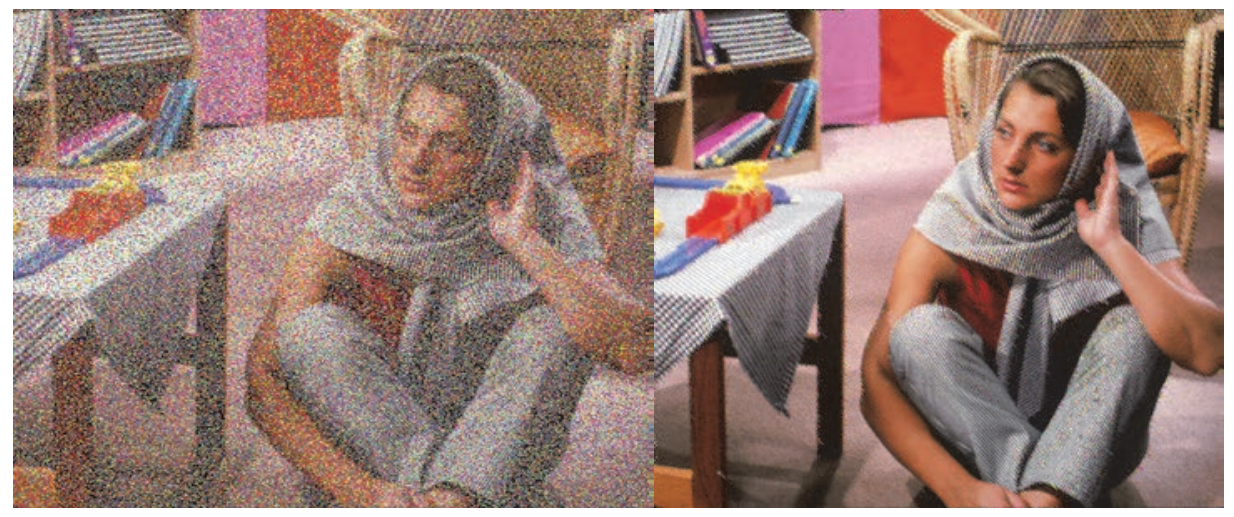

Fig. 8. Results obtained for the noise level (30\%) corrupted for the 512x512 Barbara color image and its denoised image. 


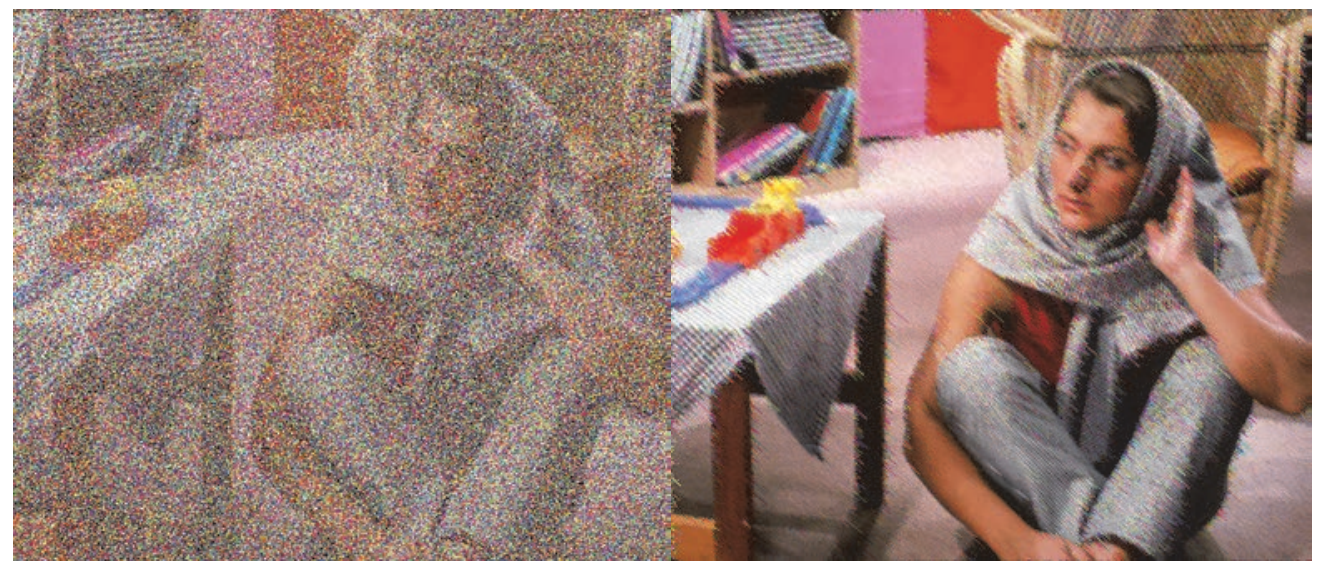

Fig. 9. Results obtained for the noise level (60\%) corrupted for the 512x512 Barbara color image and its denoised image.

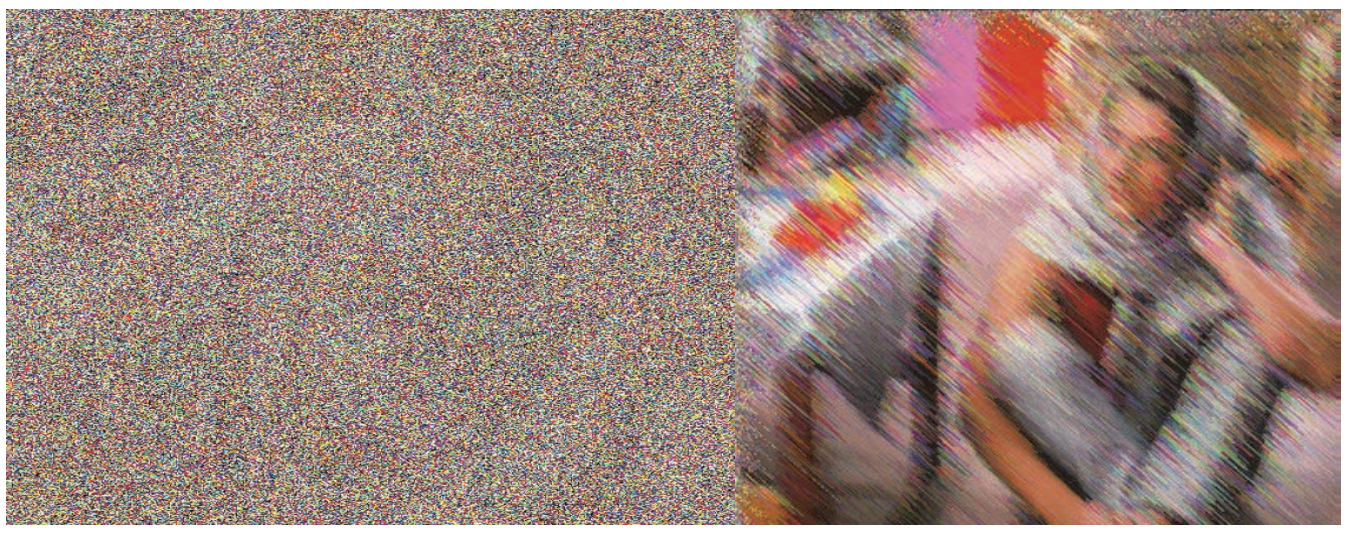

Fig. 10. Results obtained for the noise level (90\%) corrupted for the 512x512 Barbara color image and its denoised image.

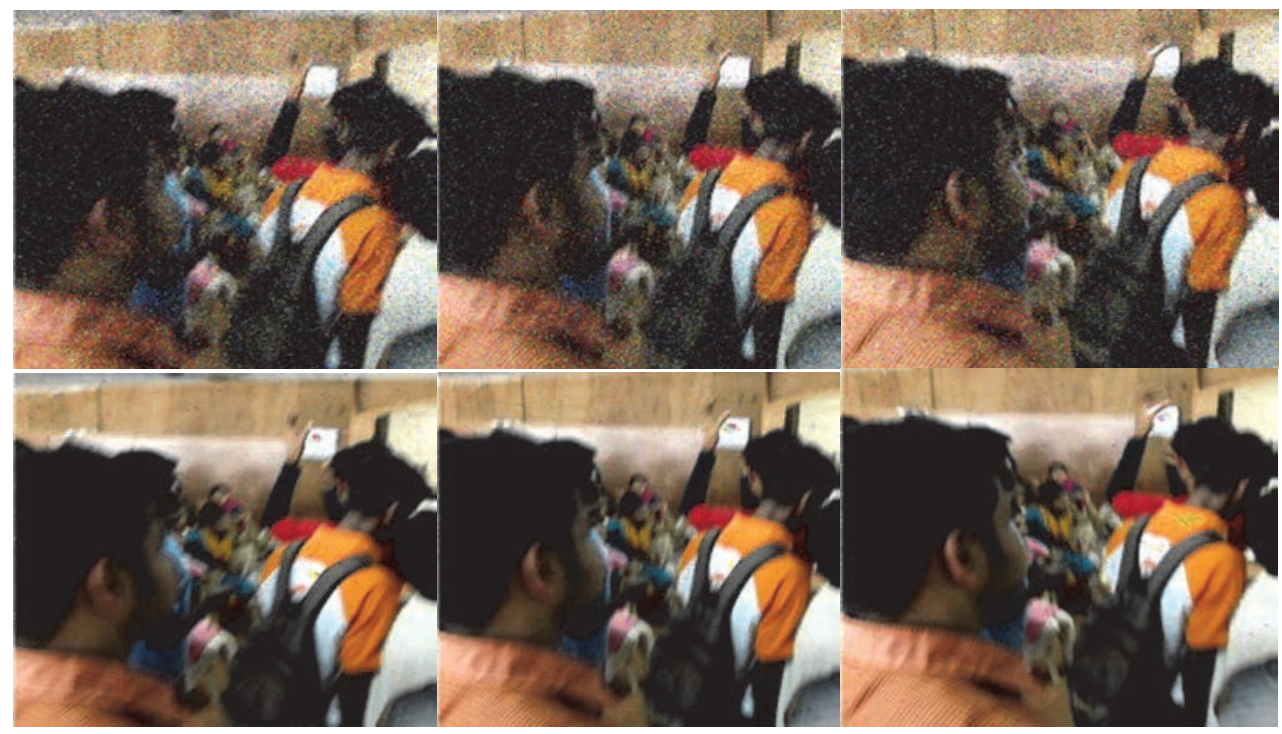

Fig. 11. Results obtained for the noise corrupted video frames with $10 \%$ noise density and its corresponding denoised frames (top threeframes are noise frames and corresponding bottom frames are denoised frames). 


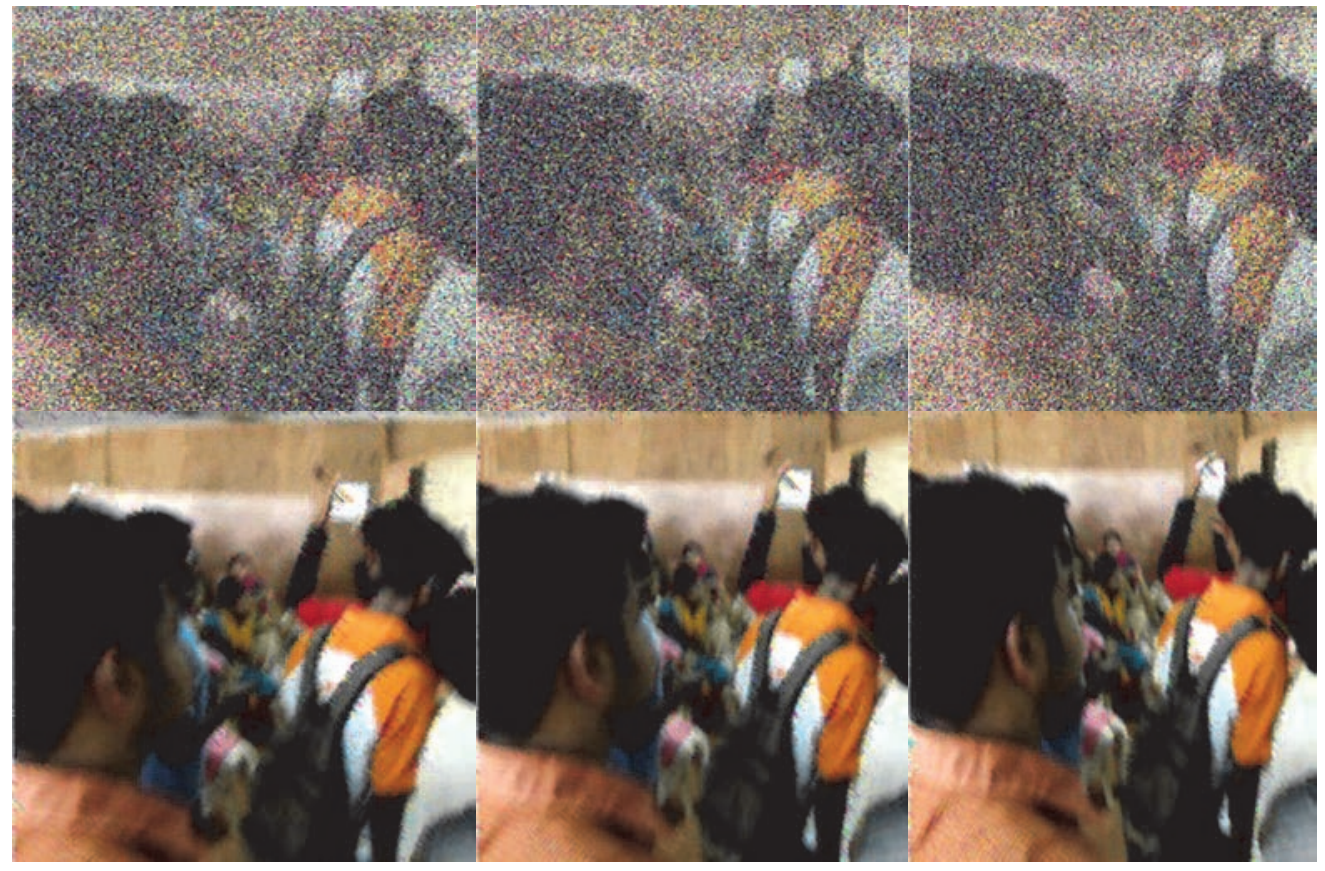

Fig. 12. Results obtained for the noise corrupted video frames with $60 \%$ noise density and its corresponding denoised frames (top three frames are noise frames and corresponding bottom frames are denoised frames).

The performance of the proposed algorithm is tested for various levels of noise corruption and compared with standard filters namely standard median filter (SMF) and weighted median filter (WMF). The performance of the developed algorithm and other standard algorithms are quantitatively measured by the following parameters such as peak signal-to-noise ratio (PSNR), mean square error (MSE) and structural similarity (SSIM) index. They are defined by the following Eqs. 1-3.

where

$$
\mathrm{PSNR}=10 \log _{10}\left(255^{2} / \mathrm{MSE}\right) \text {, }
$$

$$
M S E=\frac{1}{(m * n * 3)} \sum_{x=1}^{m} \sum_{y=1}^{n}\left(f(x, y)-f^{\prime}(x, y)\right),
$$

$\mathrm{M}$ and $\mathrm{N}$ are the total number of pixels in the horizontal and the vertical dimensions of the image, $f$ and f' denote the original and filtered image respectively.

$$
\operatorname{SSIM}(x, y)=\frac{\left(2 \mu_{x} \mu_{y}+c_{1}\right)\left(2 \sigma_{x y}+c_{2}\right)}{\left(\mu_{x}^{2}+\mu_{y}^{2}+c_{1}\right)\left(\sigma_{x}^{2}+\sigma_{y}^{2}+c_{2}\right)} .
$$

Where, $\mu_{x}$ is the average of $x, \mu_{y}$ is the average of $y, \sigma_{x}{ }^{2}$ is the variance of $x, \sigma_{y}{ }^{2}$ is the variance of $y, \sigma_{x y}$ is the covariance of $x$ and $y \cdot c_{1}=\left(k_{1} L\right)^{2}, c_{2}=\left(k_{2} L\right)^{2}$ are the two variables to stabilize the division with weak denominator. Where $\mathrm{k}_{1}=0.01$ and $\mathrm{k}_{2}=0.03$ by default. $L$ is the dynamic range of the pixel-vales (2 \#bits per pixel-1).

Tables 2 and 3 shows the comparison of PSNR and MSE values with different algorithms like the standard median filter (SMF), adaptive median filter (AMF) and decision based method (DBM). The comparison shows better performance of the proposed lone diagonal sorting algorithm (LDS) in terms of PSNR and MSE. Table 4 gives computational time for various noise densities for Lena color image of size (512x512).

Table 2. Comparative results of various filters for $30 \%$ corrupted Barbara image.

\begin{tabular}{lrrrc}
\hline METRIC & \multicolumn{1}{c}{ SMF } & AMF & \multicolumn{1}{c}{ DBM } & Proposed LDS \\
\hline MSE & 268.74 & 94.94 & 100.98 & 54.68 \\
PSNR & 23.84 & 28.36 & 28.09 & 30.75 \\
\hline
\end{tabular}

Table 3. Comparative results of various filters for $90 \%$ corrupted parrot image.

\begin{tabular}{lcccc}
\hline METRIC & SMF & AMF & DBM & Proposed LDS \\
\hline MSE & 9699.2 & 1264.2 & 812.77 & 798.0313 \\
PSNR & 8.26 & 17.11 & 19.03 & 19.12 \\
\hline
\end{tabular}


Table 4. Computational time for denoising Lena color (512x512) image.

\begin{tabular}{lcc}
\hline NOISE & $\begin{array}{c}\text { TIME (sec) } \\
\text { LDS }\end{array}$ & $\begin{array}{c}\text { TIME (sec) } \\
\text { SMF }\end{array}$ \\
\hline 0.1 & 26 & 38 \\
0.2 & 27 & 39 \\
0.3 & 28 & 40 \\
0.4 & 28 & 40 \\
0.5 & 28 & 40 \\
0.6 & 28 & 40 \\
0.7 & 28 & 40 \\
0.8 & 28 & 40 \\
0.9 & 28 & 40 \\
\hline
\end{tabular}

\section{CONCLUSION}

A robust yet efficient algorithm to remove impulsive noise from color images and video sequences is proposed. The lone diagonal sorting algorithm clearly reduces the computational time required for denoising the corrupted images because of the lower number of computations required as compared to other standard algorithms. Also since the noisy pixels are replaced with the immediate neighborhood pixels, the image quality is comparatively appreciable. The lower number of computations performed due to the diagonals sorting alone whereas other standard algorithms involve many comparisons and computations there by our algorithm clearly reduces the hardware complexity, thereby increasing the efficiency and speed of operation and also reducing the cost of the system without compromising much on the quality of the images and videos when compared with other competitive algorithms. This proposed algorithm is tested with Barbara and Parrot color images for various noise densities ranging from $10 \%$ to $90 \%$ and for the video signal ranging from $10 \%$ to $60 \%$ and it gives good results. Further this work might be extended for the impulse noise removal considering random noise such as the noises with one or two percent tolerance with salt and pepper noise.
This noise removal technique further could be extended for the three dimensional color images.

\section{REFERENCES}

Aiswarya K, Jayaraj V, Ebenezer D (2010). A new and efficient algorithm for the removal of high density salt and pepper noise in images and videos, 2nd Int Conf Computer Modeling and Simulation, 22-24 January, 2010, Sanya, China. ICCMS 4:409-13.

Astola J, Kuosmanen P (1997). Fundamentals of non-linear digital filtering. Boca Raton: CRC Press; New York: John Wiley\& Sons.

Chan RH, Ho CW, Nikolova M (2005). Salt and pepper noise removal by median type noise detectors and detail preserving regularization, IEEE Transactions on Image Processing 14:1479-85.

Gallagher NC, Wise GL (1981). A theoretical analysis of the properties of median filters. IEEE Trans Acoust Speech and Signal Processing, ASSP - 29:1136-41.

Gonzalez RC, Woods RE (2008). Digital image processing: Pearson International Edition.

Jeako S, Lee YH (1991). Center weighted median filters and their applications to image enhancement, IEEE Trans Circuits and Systems 38:984-93.

Luo W (2006a). An efficient detail-preserving approach for removing impulse noise in images. IEEE Signal Process Lett 13:413-6.

Luo W (2006b). Efficient removal of impulse noise from digital images. IEEE Transactions on Consumer Electronics 52:523-7.

Nodes TA, Gallagher NC (1987). Median filters: some modifications and their properties. IEEE Trans Acoust, Speech and Signal Processing ASSP 30:739-46.

Pitas I, Venetsanopoulous AN (1990). Non-linear digital filter principles and applications, Boston: Kluwer academic publishers.

Pratt WK (1978). Digital image processing. New York: John Wiley\& Sons.

Ratna Babu K, Arun Rahul L, Vineet Souri P, Suneetha A (2011). Image denoising in the presence of high level salt and pepper noise using modified median filter, Int J Computer Science and Technology 2:80-3. 